\title{
Cuentas energéticas no habituales en edificación residencial
}

\section{Towards new energy accounting in residential building}

\author{
M. de Luxán García de Diego ${ }^{(*)}$, G. Gómez Muñoz ${ }^{(* *)}$, E. Román López ${ }^{(* *)(*)}$
}

\section{RESUMEN}

La evaluación energética de edificios, suele cuantificar resultados referentes a consumos, ahorros energéticos y económicos, amortizaciones, emisiones de $\mathrm{CO}_{2}$, etc.; sin embargo, existen otros factores, habitualmente no considerados, que pueden ser determinantes en la elección de soluciones para el mejor comportamiento y la eficiencia energética global de las edificaciones y en el bienestar y la calidad de vida de sus usuarios. En el presente artículo se muestran casos reales en los que aparecen ejemplos y cuestiones, como la inadecuación de algunos parámetros en condiciones sociales de pobreza energética donde no cabe valorar ahorros sin consumos, la interrelación entre indicadores energéticos y otros temporales y sociales habitualmente poco tratados, la influencia de soluciones constructivas que se contabilizan energéticamente de modo poco efectivo, pero que consiguen resultados reales superiores, las variaciones en los modelos de confort, y la atención a las estrategias teniendo en cuenta los escenarios del cambio climático.

Palabras clave: Rehabilitación energética; eficiencia energética; cambio climático; pobreza energética.

\section{ABSTRACT}

The energy assessment of buildings usually quantifies information concerning energy consumption and savings and its economic counterparts, rates of return, $\mathrm{CO} 2$ emissions, etc. However, there are other factors often not considered that may be decisive when selecting solutions for the best performance and overall energy efficiency of buildings and the quality of life of its dwellers. In this paper, we show real cases exemplifying subjects like: the inadequacy of certain parameters under energy poverty social conditions where it makes no sense studying savings without any consumption, the interrelationship of energy indicators with other socially and time related scarcely dealt with, the influence of constructive solutions with a poor energy efficiency assessment that achieve superior real results, the variations in the comfort models and the strategies designed taking in account climate change scenarios.

Keywords: Energy retrofitting; energy efficiency; climate change; energy poverty.

(*) Universidad Politécnica de Madrid. Madrid (España).

(**) cc6o Estudio de Arquitectura S.L.P. Madrid (España).

Persona de contacto/Corresponding author: mrgluxan@telefonica.net (M. de Luxán García de Diego)

Cómo citar este artículo/Citation: Luxán García de Diego, M. d., Gómez Muñoz, G., Román López, E. (2015). Cuentas energéticas no habituales en edificación residencial. Informes de la Construcción, 67(EXTRA-1): mo28, doi: http://dx.doi.org/10.3989/ic.14.059.

Licencia/License: Salvo indicación contraria, todos los contenidos de la edición electrónica de Informes de la Construcción se distribuyen bajo una licencia de uso y distribución Creative Commons Reconocimiento no Comercial 3.o. España (cc-by-nc). 


\section{INTRODUCCIÓN}

\subsection{Antecedentes}

La evaluación energética de edificios suele cuantificar consumos, ahorros energéticos y económicos, amortizaciones, emisiones de $\mathrm{CO}_{2}$, etc.; sin embargo, existen otros factores, habitualmente no considerados, que pueden ser determinantes en la elección de soluciones para el mejor comportamiento y la eficiencia energética de los edificios y en el bienestar y la calidad de vida de sus usuarios, especialmente en las viviendas. Estos elementos, que conducen a una contabilidad energética no habitual, suelen estar más relacionados con el comportamiento de los habitantes, con aspectos sociales y con cuestiones ajenas al sector residencial, que con el comportamiento energético de los edificios.

Los estudios realizados basados en encuestas (1) o en monitorización de edificios (2) muestran que los consumos reales de energía en los hogares suelen ser diferentes a los previstos en las simulaciones energéticas. Las razones son diversas, desde los estilos de vida de las personas y ocupación de las viviendas diferentes a los perfiles previstos, hasta razones asociadas a aspectos económicos y sociales. Por ello, sería necesario revisar los métodos de cuantificación energética que evalúan las medidas de mejora sobre los edificios, para incorporar aquellos elementos que permitan aumentar el conocimiento sobre el comportamiento real del parque edificado. De esta manera, las soluciones elegidas se adaptarán mejor a las necesidades de los usuarios. A continuación se describirán algunos de estos factores.

\section{EL AHORRO DE ENERGÍA FRENTE LAS CONDICIONES ECONÓMICAS DE LOS USUARIOS}

Habitualmente, cuando se habla de actuaciones en la construcción para reducir su consumo energético se obvia una cuestión fundamental como es el confort interior alcanzable en relación a las posibilidades reales de los habitantes y su capacidad de bienestar. Este tema es fundamental si las viviendas se sitúan en zonas climáticas que requieren un aporte de energía para conseguir condiciones interiores adecuadas.

Por tanto, las actuaciones en los edificios, tanto nuevos como existentes, deberían dirigirse al ahorro en el consumo energético y la disminución de emisiones en relación a la mejora de las condiciones de habitabilidad, incidiendo en todos aquellos aspectos que confluyen para garantizar un mejor y equilibrado modo de vida para los usuarios.

Si se analiza el consumo de energía de un edificio se comprueba que la mayor parte se produce en su climatización a lo largo de su funcionamiento. Este factor adquiere gran importancia cuando se combina un uso intensivo de las instalaciones, en muchas ocasiones poco eficientes, con una envolvente térmica también ineficiente desde el punto de vista energético.

En esta situación se encuentra más de la mitad del parque inmobiliario en España, pues fue construido antes de la aparición de la primera normativa específica para la reducción de la demanda energética de los edificios en 1979.

$\mathrm{Si}$ a estos antecedentes se les añade el aumento de los precios de la energía en los últimos años y la disminución de los ingresos en los hogares, derivada de la crisis económica, nos encontramos con gran cantidad de usuarios que son incapaces de satisfacer sus necesidades básicas de confort porque no pueden pagar las facturas energéticas. Recordemos que esta circunstancia de "pobreza energética», concepto que fue definido en Gran Bretaña en 1991 (3) comprende a los consumidores que destinan un porcentaje mayor del $10 \% \mathrm{de}$ sus ingresos familiares a pagar las facturas de energía de su vivienda. A esta situación actualmente se enfrentan más de 50 millones de personas en Europa.

Según el informe presentado en marzo de 2014 por la Asociación de Ciencias Ambientales sobre la pobreza energética en España, la situación en nuestro país ha empeorado en los últimos años, revelándose datos preocupantes respecto a los ofrecidos por el informe realizado dos años antes: «En 2012, el porcentaje de hogares con gastos de energía desproporcionados alcanzó el $17 \%$ (más de 7 millones de personas) y el porcentaje de hogares españoles que se declaran incapaces de mantener su vivienda a una temperatura adecuada en invierno era del $9 \%$ (alrededor de 4 millones de personas). Esto significa un aumento del $34 \%$ en el primer aspecto valorado y $19 \%$ en el segundo en tan solo dos años de evolución» (4). En la Figura 1 se muestra la evolución de esta tendencia desde 2006 hasta 2012.

A estos datos habría que añadir los que se extraen de la Encuesta de Condiciones de Vida (6), donde el $6 \%$ tiene retrasos en el pago de las facturas correspondientes a la energía y el $25 \%$ de los hogares no pueden mantener una temperatura fresca en el interior de la vivienda en los meses más calurosos. Esta última circunstancia, combinada con los efectos derivados del cambio climático, hace que sea necesario replantear las estrategias de cara al futuro donde, según los datos de los estudios sobre escenarios de cambio climático realizados por la Agencia Estatal de Meteorología (AEMET) (7), aumentarán crecientemente los problemas en la refrigeración de las viviendas en los periodos más cálidos en España.

Según datos de la Comisión Nacional de la Energía, Endesa e Iberdrola en el año 2006 se registraron 600.000 cortes de energía eléctrica por impago, en el año 2012 se suspendió el suministro a 1.400.00o viviendas, el 7 \% del total de los clientes domésticos; si se mantuviese la progresión, en el 2013 se hubiese llegado al $8 \%$. Puede afirmarse que la mayoría de esos cortes se produjeron en viviendas distintas, aunque hay

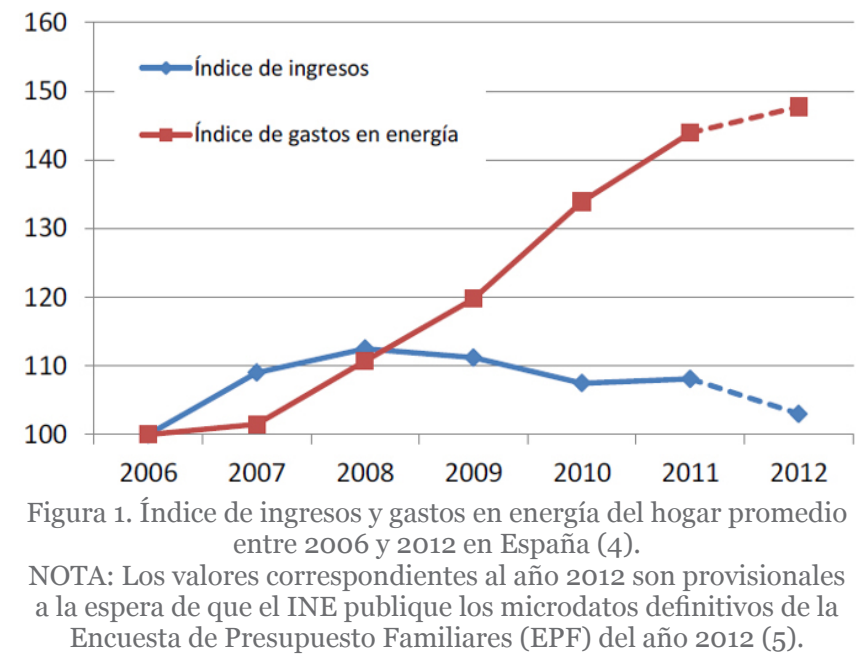


que tener en cuenta que en algunos casos se trata de segundas residencias. De estos cortes en los suministros, el $67 \%$ consigue pagar antes de que pasen 48 horas, el $11 \%$ tarda entre 48 horas y una semana y el $22 \%$ se tiene que retrasar entre una semana y un año.

Esta circunstancia ya estaba recogida en el estudio «Retrato del chabolista con piso» de 1989, (8) donde se indicaba que en los barrios de vivienda social periféricos de Madrid, el $40 \%$ de los vecinos no usaba la calefacción porque no podía pagar la energía.

En la rehabilitación energética del edificio demostrador del Proyecto LIFE + «Lucha contra el cambio climático: tecnologías innovadoras para un uso eficiente de recursos y energía en restauración de viviendas» NEWsolutions4OLDhousing (LIFE10 ENV/ES/439), en el que participamos, tras la realización de una encuesta se ha comprobado que, aunque el clima de Zaragoza es relativamente frío, el $44 \%$ de los vecinos, por sus condiciones económicas, no usan la calefacción porque no pueden pagar la factura (9) (10) (11).

Bajo estas circunstancias, si el ahorro y la eficiencia energética se miden en términos de consumo se podría llegar a la paradoja de que hablemos de grandes ahorros con determinadas actuaciones, cuando no existe previamente gasto real alguno que disminuir, debido a las circunstancias antes descritas.

Es decir, que podemos encontrar edificios en los que un porcentaje muy alto de usuarios no gastan energía porque no la pueden pagar, aunque vivan durante gran parte del año en un ambiente interior muy alejado de los parámetros básicos de confort. Esta circunstancia es característica en los centros históricos de muchas ciudades españolas, donde existen edificios patrimoniales habitados por personas mayores o con pocos recursos económicos, y con envolventes muy poco eficientes en términos energéticos, en los que habitualmente no hay instalaciones de climatización, o las que hay son muy poco eficientes.

Por ello, se reafirma el papel relevante de la reducción de la demanda de energía mediante el diseño y las soluciones pasivas de los edificios frente a otras actuaciones únicamente destinadas a la reducción del consumo mediante el cambio de las instalaciones. La intervención en la envolvente térmica del edificio y un buen diseño de los sistemas constructivos que la caracterizan, buscando el mejor aprovechamiento de las condiciones climáticas del entorno, pueden conseguir una mayor habitabilidad de las viviendas sin que sea necesario el consumo energético, que en algunas condiciones es prohibitivo para los usuarios.

En muchos casos, al hacer el balance energético y económico de las medidas de eficiencia sobre un edificio, se valoran algunos de los de sistemas energéticos activos como los de menor periodo de retorno de la inversión, ya que se compara una situación inicial de consumo frente a una posterior en la que se ha mejorado la eficiencia del sistema. Teniendo en cuenta los estudios sobre las condiciones de algunos usuarios hay que recordar que la amortización de las medidas depende del aprovechamiento y el uso que puedan hacer de ellas los habitantes; una instalación que no se usa no llega a amortizarse nunca. Sin embargo, las medidas pasivas sobre el edificio pueden ser aprovechables por todos los usuarios sea cual sea su situación económica, ya que implican una mejora del comportamiento térmico independientemente del uso de sistemas activos de climatización.

\section{INDICADORES ENERGÉTICOS INTERRELACIONADOS: OPORTUNIDAD TEMPORAL DE LA CONSTRUCCIÓN, CLIMATIZACIÓN, POBLACIÓN, EVOLUCIÓN DE LOS HOGARES}

La selección de indicadores de energía asociados al uso de las viviendas tiene enorme trascendencia en la evaluación de las medidas destinadas a reducir el consumo de energía en los edificios. En función de los resultados que ofrezcan estos indicadores, la evaluación de los resultados puede hacerse de diferente manera. En el caso de la vivienda, si se utilizan únicamente indicadores que evalúan el consumo de energía por superficie construida, los resultados pueden excluir otras cuestiones trascendentes en la toma de decisiones para la intervención sobre el parque edificado.

En España, la evolución del consumo de energía en climatización por superficie construida ha disminuido en el último medio siglo a partir de 1979, con la aplicación de la Norma Básica de la Edificación NBE-CT-79. La aprobación en 2006 del Código Técnico de la Edificación (CTE) y la revisión del documento sobre ahorro de energía en 2013 han supuesto importantes cambios en el sector de la edificación, en el que los aspectos de ahorro y eficiencia energética están adquiriendo cada vez mayor protagonismo.

Sin embargo, el estudio del conjunto de viviendas para el caso de Madrid muestra que el consumo de energía por habitante en el periodo entre 1940 y $2010^{1}$, a pesar de la aparición de estas normativas, se ha incrementado considerablemente debido, por un lado, a que no se ha intervenido masivamente en la rehabilitación energética de las viviendas construidas con anterioridad a $1979 \mathrm{y}$, por otro, al gran número de viviendas construidas en estos años en los que se han ido produciendo sucesivos «booms» inmobiliarios (12) (13). El sobredimensionamiento del parque edificado en relación a las necesidades de la población, implica necesariamente un incremento del gasto de recursos, a pesar de que a las viviendas nuevas se les exija cada vez menor consumo de energía. A esto se añade que los estándares de confort de las viviendas se han modificado desde mediados del siglo pasado, incorporándose instalaciones de calefacción y refrigeración tanto en las nuevas construcciones como en las viviendas existentes.

Los datos de la evolución del consumo de energía en climatización para el conjunto de las viviendas en Madrid $^{2}$ sirven de

\footnotetext{
${ }^{1}$ El número de viviendas construidas después de la Guerra Civil en Madrid supone cerca del 90 \% respecto a la totalidad de viviendas existentes.

${ }^{2}$ Los valores de este indicador se han obtenido teniendo en cuenta el número de viviendas construidas en Madrid en el periodo estudiado y asignando a cada una de ellas un consumo de energía en función de sus características constructivas. A partir de ahí, se obtiene un consumo medio anual en climatización ponderado según el número de viviendas de cada periodo.
} 
muestra para ilustrar esta idea. En la Figura 2 se muestra la evolución de este indicador entre 1940 y 2010, tanto por superficie como por habitante. Se observa que si el primero se redujo casi a la mitad en estas décadas, el segundo se duplicó en el mismo periodo. Únicamente se observa al final del periodo estudiado una estabilización de ambas variables, debido al brusco descenso de la construcción de viviendas por la crisis económica.

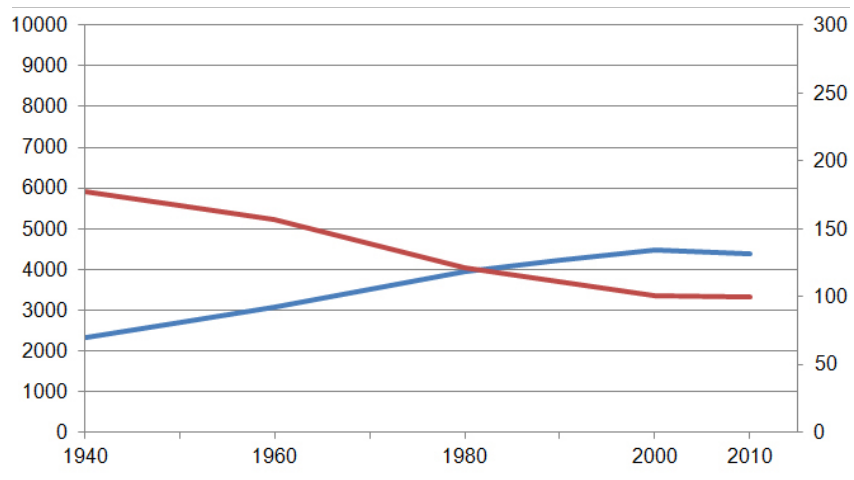

—Consumo anual de energía en climatización por habitante (kWh/hab) / Eje izquierdo —Consumo anual de energía en climatización por superficie (kWh/m2) / Eje Derecho Figura 2. Evolución del consumo anual de energía en climatización de viviendas en Madrid entre 1940 y 2010. Fuente: G. Gómez (14).

Estos datos se han elaborado a partir de la consideración del conjunto de todas las viviendas construidas en Madrid en ese periodo, sin discriminar aquellas vacías o de segunda residencia. Por tanto, es posible que al considerar los datos reales de consumo, los valores de ambos indicadores disminuyan, pero seguramente no así las tendencias de cada uno de ellos.

Esta disparidad de tendencias entre un indicador de consumo de energía por superficie y otro por habitante se hace más notable cuando, además de la energía destinada a climatización, se considera la totalidad del proceso constructivo, es decir, la energía también en la construcción y la producción de las viviendas.

En la Figura 3 se observa que los valores de los indicadores que tienen en cuenta estas fases del proceso constructivo son mayores que en el ejemplo anterior y que, en el caso del consumo anual de energía por habitante, el incremento en el periodo 1940 y 2010 es mayor, ya que se imputan en el cálculo todos los recursos energéticos utilizados en la construcción y el uso de las edificaciones.

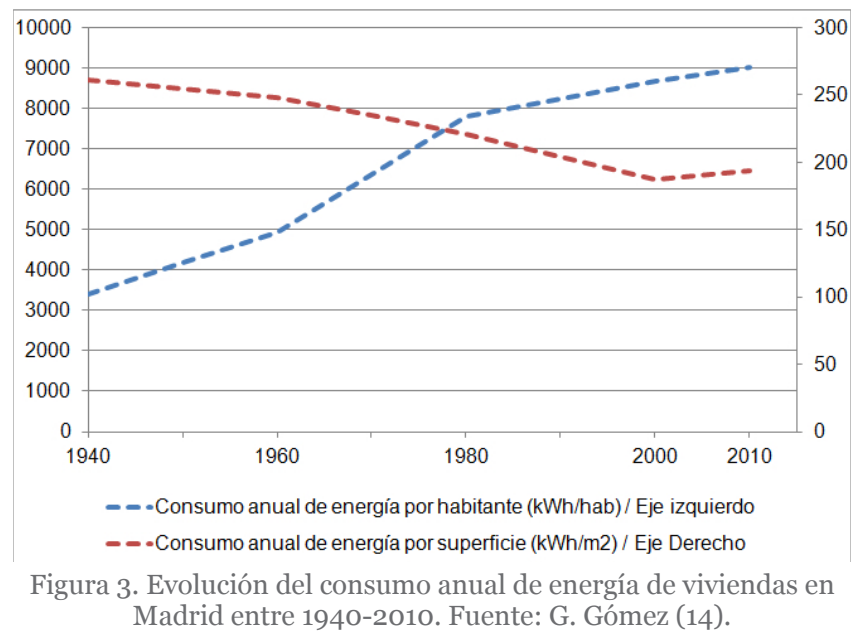

Si complementamos esta información con los datos de viviendas nuevas construidas en el periodo 1940-2010, frente a las viviendas rehabilitadas energéticamente, establecidos en una proporción de 20 a 1 (15), es fácil llegar a la conclusión de que este efecto en el incremento del consumo por habitante se debe a la intensa actividad constructora de nueva planta.

Por otro lado, estas estimaciones muestran que, si bien el consumo de energía habría disminuido por la mejora de la calidad energética de las edificaciones entre 1940 y el 2010, la construcción de un gran número de viviendas, aunque fueran más eficientes, supondría un incremento considerable en el consumo total de energía por estas nuevas unidades. Si el número de habitantes se mantiene en las próximas décadas, una política destinada a la rehabilitación energética de las edificaciones, en lugar de a la sustitución de edificios, supondría una importante reducción del consumo anual de energía destinada al uso y mantenimiento de las viviendas. No hay que olvidar que rehabilitar un edificio de viviendas, aunque se sustituyan todas las carpinterías, se le dote de aislamientos y se le cambien las instalaciones, supone un ahorro energético y de contaminación del $60 \%$ aproximadamente frente a la construcción de otro nuevo equivalente, si tenemos en cuenta la energía contenida en los materiales (16). Si esta cuestión se traslada a la totalidad de las viviendas existentes, la escala de intervención se incrementa, mientras que la magnitud de los impactos ambientales asociados disminuye mediante la implementación de políticas que fomenten la rehabilitación frente a la demolición y sustitución (17).

Otra cuestión que puede ser relevante es la relación entre la dimensión de las viviendas y el número de habitantes que las utilizan. En ese sentido, la transformación del parque de viviendas en función de la composición de los grupos y tipos de familias puede suponer importantes reducciones del consumo de recursos para la climatización de las mismas. Los estudios sobre la evolución de los hogares en la Comunidad de Madrid indican la reducción del número de sus miembros para las próximas décadas (18). La intervención sobre las edificaciones existentes debe tener en cuenta estas cuestiones, de manera que a largo plazo puede ser mucho más eficaz, en la reducción de los impactos ambientales, una rehabilitación energética que tenga en cuenta propuestas para la división o unificación de las viviendas en función de las características de sus ocupantes.

Por tanto, de cara a la reducción a largo plazo del impacto ambiental asociado al uso de las viviendas, convendría tener en cuenta no solo indicadores de energía o contaminación atmosférica, sino otros índices de carácter relativo, por ejemplo, a la oportunidad temporal en la construcción y a la evolución y composición de la población, de manera que sea posible relacionar diferentes aspectos que finalmente influyen en la cuantificación de los recursos necesarios para el funcionamiento de la ciudad.

\section{ESTRATEGIAS DE DISEÑO PASIVO EN LAS VIVIENDAS: SIMULACIONES DESALENTADORAS Y ESTIMACIONES POSITIVAS}

En diversas publicaciones y programas de simulación, se avanzan cuantificaciones muy desalentadoras sobre la efica- 
cia y, sobre todo, el retorno económico de la aplicación de medidas pasivas que, sin embargo, parecen evidentemente positivas desde el sentido común. Como se ha señalado anteriormente, este tipo de medidas tienen un impacto directo sobre el bienestar de los usuarios, sin que ello suponga consumo de energía durante su uso.

Como ejemplo, en un caso de incorporación de protecciones solares pasivas, si se comparan los datos previstos teóricamente y los reales, se extraen conclusiones de interés para describir esta idea.

Para esta comparación, entre varias publicaciones coincidentes en sus apreciaciones sobre las estimaciones de ahorro de energía en función de las medidas de intervención previstas, se ha seleccionado una publicación sobre «Eficiencia energética en la rehabilitación de edificios» (19) que, con vocación de guía de recomendaciones, se basa en la utilización de simulaciones energéticas con herramientas conocidas (LIDER, CALENER, Energy Plus) así como estudios publicados por IDAE (1) para Sevilla, Barcelona, Madrid y Burgos.

En este estudio, según las simulaciones realizadas, una medida que implementa «Protecciones solares pasivas con elementos móviles como lamas motorizadas o persianas», aplicable a viviendas, es eficaz, ya que en zonas menos calurosas que Sevilla la disminución de la energía de refrigeración podría llegar al $40 \%$. Sin embargo concluye que, como el gasto en refrigeración es escaso, en el caso de zonas más frías el ahorro es «insignificante». Para Madrid indica que el periodo de retorno de la inversión en protecciones solares en viviendas es de más de 100 años.

Sin embargo, el estudio de un caso real ha llegado a otra conclusión. La acción consistió en la colocación de persianas exteriores enrollables en miradores acristalados en un edificio de viviendas en la zona de Arturo Soria, en Madrid33. En la Tabla 1 se recogen los consumos y los gastos en refrigeración reales. La colocación de las persianas se realizó durante el verano del 2010:

El consumo medio de electricidad en refrigeración en los años previos es de $12.757 \mathrm{kWh} /$ año, y en los posteriores de $7.457 \mathrm{kWh} / \mathrm{año}$, con lo que se ha conseguido un $42 \%$ de ahorro energético medio. El precio del recibo eléctrico ha aumentado en los 5 años medidos un $64 \%$, lo que supone aproximadamente un $13 \%$ anual.

El coste de la instalación de las protecciones solares en los miradores acristalados del edificio ha sido de $16.800 €$. Si el precio de la electricidad se mantiene con el mismo incremento, las protecciones se amortizarían, contando sólo con el ahorro en refrigeración, en menos de 7 años. Este valor está muy alejado de la cuantificación prevista teóricamente en el estudio mencionado.

Realmente se amortizaría aún en menos tiempo, ya que también tienen un resultado positivo en el ahorro energético en calefacción si se cierran durante las noches de los meses fríos, pero no contamos con datos de consumos energéticos segregados sobre esa circunstancia para poder hacer afirmaciones específicas.

La revisión de las recomendaciones derivadas de cuantificaciones de la eficacia de las medidas pasivas en nuestros climas es urgente, sobre todo las que se relacionan con las que puedan ayudar a la refrigeración. Para ello, es necesario contrastar las herramientas de simulación energética y económica con el estudio de casos reales, para no producir un efecto desalentador sobre ciertas medidas que, en la realidad, son muy positivas de cara al ahorro energético, a la mejora del confort y en los periodos de amortización, pero que en las simulaciones salen claramente infravaloradas y perjudicadas.

\section{ESTIMACIÓNES DE CONSUMO DE ENERGÍA EN VIVIENDA FRENTE A LOS MODELOS DE CONFORT}

La evaluación del confort térmico en el interior de un edificio es una tarea compleja, para la que existen diversos métodos normalizados pues, además de las variables cuantificables que influyen directamente sobre esta sensación, como son la temperatura, la humedad relativa, la velocidad del aire, la actividad física y el arropamiento, existe una componente subjetiva, que en muchos casos es función de las costumbres y características metabólicas y físicas de las personas. Sin embargo, la definición del confort es fundamental en la cuantificación de la energía que requieren los edificios.

De forma general, los métodos para determinar el confort, y por tanto, las necesidades de climatización se pueden clasificar en dos categorías: cuantitativos y cualitativos.

Los modelos cuantitativos se basan en el planteamiento de que el confort higrotérmico del ser humano se produce cuando existe un equilibrio entre las ganancias y pérdidas térmicas con el entorno, y tienen en cuenta los procesos fisiológicos de regulación que dan respuesta a la radiación, la temperatura, la humedad relativa, el movimiento del aire y el arropamiento, sin considerar la capacidad metabólica de los usuarios de acomodarse a los cambios del ambiente. Algunos autores señalan la disparidad de estos modelos con estudios experimentales sobre el confort de personas reales en ambiente concretos (20). Estas diferencias parecen ser realmen-

Tabla 1. Datos anuales de consumos y gastos en energía eléctrica para refrigeración de un edificio de viviendas en la zona de Arturo Soria, Madrid.

\begin{tabular}{|l|c|c|c|c|c|}
\hline & $\mathbf{2 0 0 8}$ & $\mathbf{2 0 0 9}$ & $\mathbf{2 0 1 0}$ & $\mathbf{2 0 1 1}$ & $\mathbf{2 0 1 2}$ \\
\hline $\begin{array}{l}\text { Consumo eléctrico Refrigeración kWh/ } \\
\text { año }\end{array}$ & 11747 & 13767 & 9910 & 7365 & 7549 \\
\hline Gasto económico Refrigeración € /año & 2443 & 3084 & 2416 & 2706 & 2585 \\
\hline Coste total recibo/kWh año & 0,208 & 0,224 & 0,243 & 0,367 & 0,342 \\
\hline
\end{tabular}

Fuente: M. de Luxán

\footnotetext{
${ }^{3}$ Los arquitectos que realizan la prescripción son G. Vizcaíno y C. Marinas, los datos los han aportado la Propiedad y los responsables del mantenimiento del inmueble.
} 
te significativas en situaciones en las que los usuarios no están permanentemente en condiciones artificiales, esto es, con climatización continua y en las que se utilizan técnicas pasivas como la ventilación natural para el acondicionamiento.

Para dar respuesta a este fenómeno se han desarrollado los llamados modelos cualitativos que no reducen el confort térmico a la resolución de una ecuación de intercambio de calor, sino que pretenden tener en cuenta otros factores del usuario, físicos y psíquicos. Se basan en trabajos de campo y encuestas realizadas en diversas condiciones climáticas a partir del siguiente principio «Si se producen cambios que provocan una situación de disconfort, las personas reaccionan de forma que restauran el confort perdido» (21).

Parece, por tanto, que existe una diferente respuesta en relación al confort entre los usuarios de edificios con climatización controlada mediante sistemas convencionales frente a usuarios de edificios acondicionados con sistemas pasivos que se pueden controlar.

La utilización de un modelo de confort adaptativo permitiría a los proyectistas estimar la temperatura en la que más probablemente los usuarios del edificio alcanzaran el confort, ampliándose la zona de confort permisible, y por tanto reduciendo la necesidad de utilizar sistemas activos de climatización. El rango de temperaturas en que se estima es posible esta adaptación por parte del usuario es de $\pm 2^{\circ} \mathrm{C}$. Si además a los habitantes se les da la posibilidad de controlar las estrategias pasivas de climatización como la activación de sistemas de ventilación natural, captación y protección solar, este rango puede aumentar. Por el contrario, la sensación de disconfort aumenta si esta capacidad de control no se produce o es ineficaz, inapropiada o inutilizable.

Estas conclusiones son fundamentales, especialmente cuando se relacionan con las condiciones de ciertos usuarios que, como se ha descrito anteriormente, no pueden hacer frente al coste monetario que supone climatizar su vivienda. En estos casos el uso de sistemas pasivos es fundamental para alcanzar la habitabilidad en la vivienda.

La aplicación de medidas de diseño pasivo en los edificios supone que los elementos de la envolvente térmica estén adaptados a las condiciones del clima. Una intervención en las edificaciones que tenga en cuenta estas medidas, supone una situación de confort más flexible. En el caso del uso residencial, esta diferenciación puede resultar sustancial en algunos climas respecto a la eliminación o reducción de instalaciones o uso de aparatos de climatización que puedan necesitar energía para su funcionamiento. Según los estudios sobre confort mencionados, en los edificios climatizados las condiciones interiores no varían con el mismo ritmo que las exteriores debido a la intervención de los sistemas. Esta cuestión puede actuar en contra de la capacidad de adaptación de los usuarios, ya que no se tiene en cuenta el tiempo y los periodos de cambio de una situación a otra y suponen un mayor consumo de energía a pesar de que se utilicen aparatos de alta eficiencia.

En el caso de las viviendas situadas en nuestros climas, esta situación suele darse, sobre todo en condiciones de verano, ya que la mayor parte de las viviendas no cuentan con sistemas de refrigeración. Sin embargo, el número de viviendas con aire acondicionado se duplicó entre 1991 y 2001 (13), señalando una tendencia creciente en el uso de sistemas de refrigeración que implica el incremento del consumo de electricidad.

En relación a la necesidad de revisar los modelos de confort para tener en cuenta la influencia del diseño pasivo y la capacidad de los individuos de adaptarse a los cambios, se puede hacer referencia a algunos estudios sobre el futuro consumo energético de las viviendas considerando las modificaciones del clima (22). Varias investigaciones realizadas en el Reino Unido (23) inciden en la necesidad de profundizar en los métodos de definición del confort desde el enfoque adaptativo y en el desarrollo de estrategias pasivas que permitan a los edificios condiciones de confort no solo en la actualidad sino también en escenarios de futuro de más de 70 años.

En el caso del Reino Unido, las perspectivas de cambio climático indican un incremento de temperaturas que puede implicar la utilización de sistemas de refrigeración, que hasta este momento no han sido necesarios. En caso de prever sistemas pasivos para paliar estas condiciones, se produciría un aumento del consumo de energía en verano, aunque, por otro lado, el consumo de energía en invierno se reduciría. Holmes y Hacker han estimado que para el Reino Unido esta reducción de consumo en calefacción puede alcanzar más de un $60 \%$ en 2080 frente a los valores de 1989.

Los estudios que describen escenarios de cambio climático apuntan al incremento de temperaturas (7) (24) y con esta situación puede resultar fundamental la definición de los límites de confort para el caso del sobrecalentamiento de la época estival. Los estudios de Holmes y Hacker estiman que la temperatura superior admisible de confort en función de estos escenarios de cambio climático puede variar en el Reino Unido en torno a un $10 \%$ a lo largo de todo el año. Según estos estudios, las variaciones en los meses cálidos respecto a la situación actual parecen ser mayores, por lo que en nuestro caso, con climas más cálidos, el impacto de esta variación de rangos de confort puede ser más significativo que las predicciones para los países del norte de Europa.

Por su influencia en el consumo de energía para el uso de las viviendas, el cambio climático es una condición a considerar si se quiere reducir la energía necesaria para mantener el confort. Asimismo, el modelo de confort que se utilice para determinar las demandas de energía puede tener una gran relevancia para evaluar las medidas a implementar sobre las viviendas.

Sería necesario estudiar en qué medida ambas cuestiones pueden afectar a las edificaciones de nuestro país, considerando las condiciones propias de cada territorio y de las viviendas y usuarios que los habitan.

\section{EL CONSUMO DE ENERGÍA A LARGO PLAZO: ESCENARIOS DE CAMBIO CLIMÁTICO}

Para analizar el comportamiento térmico de un edificio normalmente se usan unos datos de un escenario climático donde las condiciones de partida son las pertenecientes a un periodo previo pasado. En el caso de España, los datos utilizados en el primer documento DB-HE1 del Código Técnico de la Edificación (2006), se basaban en diez años de recogida 
de datos y, por lo tanto, los más actualizados de aquellos corresponderían al periodo de 1995-2005 y cuyas medias serían, aproximadamente, las características del año 2000. En la actualización del documento en 2013, se ha mejorado en parte la calidad de estos datos, ajustando las zonas climáticas a situaciones más reales.

Sin embargo, desde la perspectiva de la sostenibilidad y la reducción de los impactos sobre el medio ambiente, cualquier intervención en un edificio, tanto de nueva planta como rehabilitado, debe poder utilizarse durante un plazo relativamente largo. En un análisis de futuro, si se considera que la contabilidad anual en el consumo de recursos energéticos en un edificio, considerando todas las fases del proceso, es según Vázquez (25):

Energía en la edificación $=\frac{\text { energía de fabricación }}{\text { vida útil }}+$ energía de uso $+\frac{\text { energía de demolición }}{\text { vida útil }}$

Puede afirmarse que el coste energético anual de las viviendas disminuye si se prolonga la vida útil ${ }^{4}$ de la edificación, ya que se amortiza en mayor medida la energía destinada a la transformación de recursos naturales para la construcción de la edificación.

Otra circunstancia a considerar es que los ahorros de energía y emisiones que se proponen para los próximos años, con datos de un clima cambiante, se quedarían obsoletos en un corto plazo de tiempo. Es necesario estimar el impacto que una cuestión tan relevante como el cambio climático tendrá en el sector de la edificación.

La Agencia Estatal de Meteorología (7) ha planteado una serie de escenarios regionalizados que serán los que previsiblemente habrá que tener en cuenta, y sobre los que haya que gestionar los proyectos de edificación que se propongan para la reducción del consumo de energía y de emisiones.

Las proyecciones de cambio climático aplicadas a la Península Ibérica indican que se ha producido, y seguirá produciéndose, un incremento de temperaturas y el aumento de la irregularidad del clima. Los estudios sobre el tema (24) señalan que los rasgos más destacados de la evolución de nuestros climas son los siguientes:

- Incremento progresivo de las temperaturas medias, con un calentamiento más acusado en verano que en invierno y un calentamiento estival superior en el interior que en las costas e islas.

- Mayor frecuencia de anomalías térmicas, en especial de las máximas estivales.

- Disminución de la precipitación, especialmente en primavera. Posible aumento invernal en el oeste y otoñal en el noreste.

- Aumento de los riesgos climáticos (lluvias torrenciales, olas de calor, sequías, etc.).
Si se consideran las necesidades de confort y las condiciones exteriores previstas para 15 años (hasta 2030 aproximadamente), fijando este periodo como mínimo y realista desde el punto de vista de posibles contratos de servicios energéticos y de vida de las instalaciones, se pueden extraer las siguientes conclusiones para valorar la eficacia de las actuaciones posibles sobre la edificación:

- Independientemente de las actuaciones que se realicen sobre los edificios para mejorar su eficiencia energética, se prevé una reducción de la demanda de energía para la calefacción en todas las localidades.

- Por el contrario, se incrementará la demanda de energía para refrigeración, que cada vez tendrá mayor peso e influencia para alcanzar las condiciones de confort, especialmente en los climas cálidos.

- Este cambio de configuración de las necesidades en la climatización de las viviendas, implica que las estrategias destinadas a impedir el calentamiento del espacio interior y de las envolventes en los meses de verano, principalmente las protecciones solares, adquirirán una mayor eficacia para reducir la demanda energética. En este sentido por la relación que tiene con la capacidad de adaptación de los usuarios, la aplicación de medidas pasivas puede ser fundamental para reducir el consumo de energía.

Un ejemplo de actuación sobre una edificación existente que tiene en cuenta la evolución del consumo de energía en las edificaciones a consecuencia del cambio climático es el demostrador asociado al proyecto LIFE NEWsolutions4OLDhousing (11). Se trata de la rehabilitación energética de un edificio de viviendas sociales en alquiler ubicado en Zaragoza que, además de las actuaciones para la reducción de la demanda a través de la mejora del comportamiento térmico de la envolvente, plantea la incorporación de unos parasoles fijos en fachada sur, que permiten el soleamiento en los meses fríos y garantizan la protección solar de la totalidad en meses cálidos. En la Figura 4 se incluye el detalle constructivo de las lamas y el efecto de sombreamiento sobre la fachada en los meses de invierno y verano. En la Figura 5 se aprecia el comportamiento del conjunto de la fachada en los meses de junio y diciembre.

Antes de la actuación, la demanda energética en refrigeración de este edificio sería de $14,4 \mathrm{kWh} / \mathrm{m}^{25}$. Después de la incorporación de protección solar en fachada sur, esta demanda se reduciría a $4,57 \mathrm{kWh} / \mathrm{m}^{26}$, lo que supone una reducción del $68 \%$.

Teniendo en cuenta las cuestiones planteadas, la mejor adecuación de una edificación se obtendría calculando las demandas previsibles y soluciones para reducir la energía destinada a la calefacción con las condiciones climáticas actuales, y las de refrigeración con las condiciones futuras.

Para estimar el impacto de estos posibles cambios, se ha simulado el comportamiento energético en tres zonas climáti-

\footnotetext{
${ }^{4}$ La vida útil de un edificio es una proyección a futuro del tiempo que dicho edificio va a existir. En función del tipo de análisis que se quiera hacer, este valor es diferentes. Para la valoración de inmuebles, la vida útil para el cálculo del valor de reemplazamiento neto es de 100 años (Orden ECO/805/2003). De cara a garantizar la estabilidad y seguridad de una estructura el CTE y la instrucción del Hormigón Armado EHE-08 consideran la vida útil de una estructura de hormigón en 50 años. Otra cuestión es la edad de un edificio, que el tiempo transcurrido desde su construcción hasta el momento actual. La edad media de las edificaciones en España está por debajo de los 50 años.

${ }^{5}$ Simulación con herramienta reconocida CEX3.

${ }^{6}$ Simulación con herramienta unificada Lider-Calener 2014.
} 

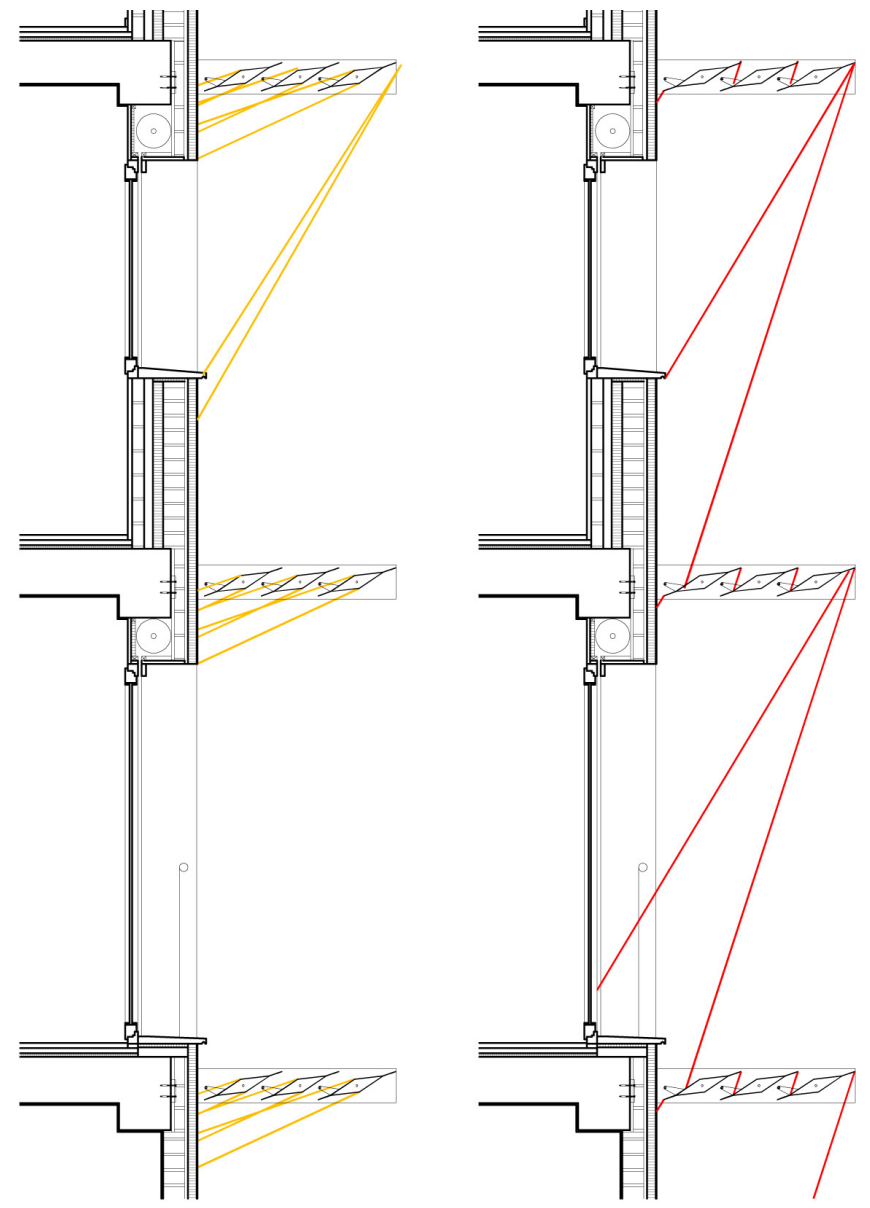

SOLEAMIENTO EN INVIERNO. 4 horas de captación solar

SOLEAMIENTO EN VERANO. 4 horas de protección solar

Figura 4. Detalle de lamas fijas orientadas en fachada sur para rehabilitación energética de edificio de viviendas (11). Fuente: Elaboración propia.

cas de un edificio de viviendas construido con anterioridad a 1979 utilizando el programa reconocido de certificación energética Ce3 según los siguientes casos:

A. Edificio en condiciones originales con datos climáticos del año 2013.

B. Edificio intervenido adaptado a las exigencias del CTE HE1 2006 y datos climáticos del 2013.

C. Edificio en condiciones originales con datos climáticos del año 2030.

D. Edificio intervenido adaptado a las exigencias del CTE HE1 2006 y datos climáticos del 2030.

Una primera conclusión de estos datos es la variación de las estimaciones en los consumos en función no solo de las actuaciones sobre el edificio, sino del cambio en las condiciones del clima. En todas las áreas cambiaría la zona climática asignada por la normativa del 2006, y por tanto la demanda de energía para alcanzar el confort.

Independientemente de las actuaciones que se realicen en la envolvente, disminuye el consumo de energía para calefacción en todos los casos. Por el contrario, el consumo de energía para refrigeración del edificio se incrementa, aunque es más significativo en los casos en los que no se prevé intervención, sobre todo la protección solar fija y móvil de huecos, y la volumetría que facilitase el sombreamiento de la envolvente.
Estos datos permiten estimar la influencia en la reducción del consumo de energía a largo plazo que pueden tener algunas actuaciones si se tiene en cuenta la modificación de las temperaturas por el fenómeno del cambio climático.

\section{CONCLUSIONES}

Las cuestiones planteadas permiten concluir que las estrategias de intervención para conseguir las mejores soluciones energéticas y de confort en la arquitectura son diferentes en función de las posibilidades sociales, del horizonte climático, o de condiciones temporales, y de una valoración lógica de las tecnologías que se manejen en cada ocasión y situación. Estas observaciones, aplicadas al conjunto del parque edificado, permitirían establecer políticas mejor equilibradas de intervención sobre las viviendas, tanto de forma inmediata como a largo plazo.

Por otro lado, la ampliación de los factores que se evalúan para determinar la eficacia de las medidas de intervención sobre las edificaciones, permitiría obtener mayor información, de manera que se pudieran orientar las intervenciones hacia actuaciones más integrales, desde una perspectiva no solo ambiental sino también temporal.
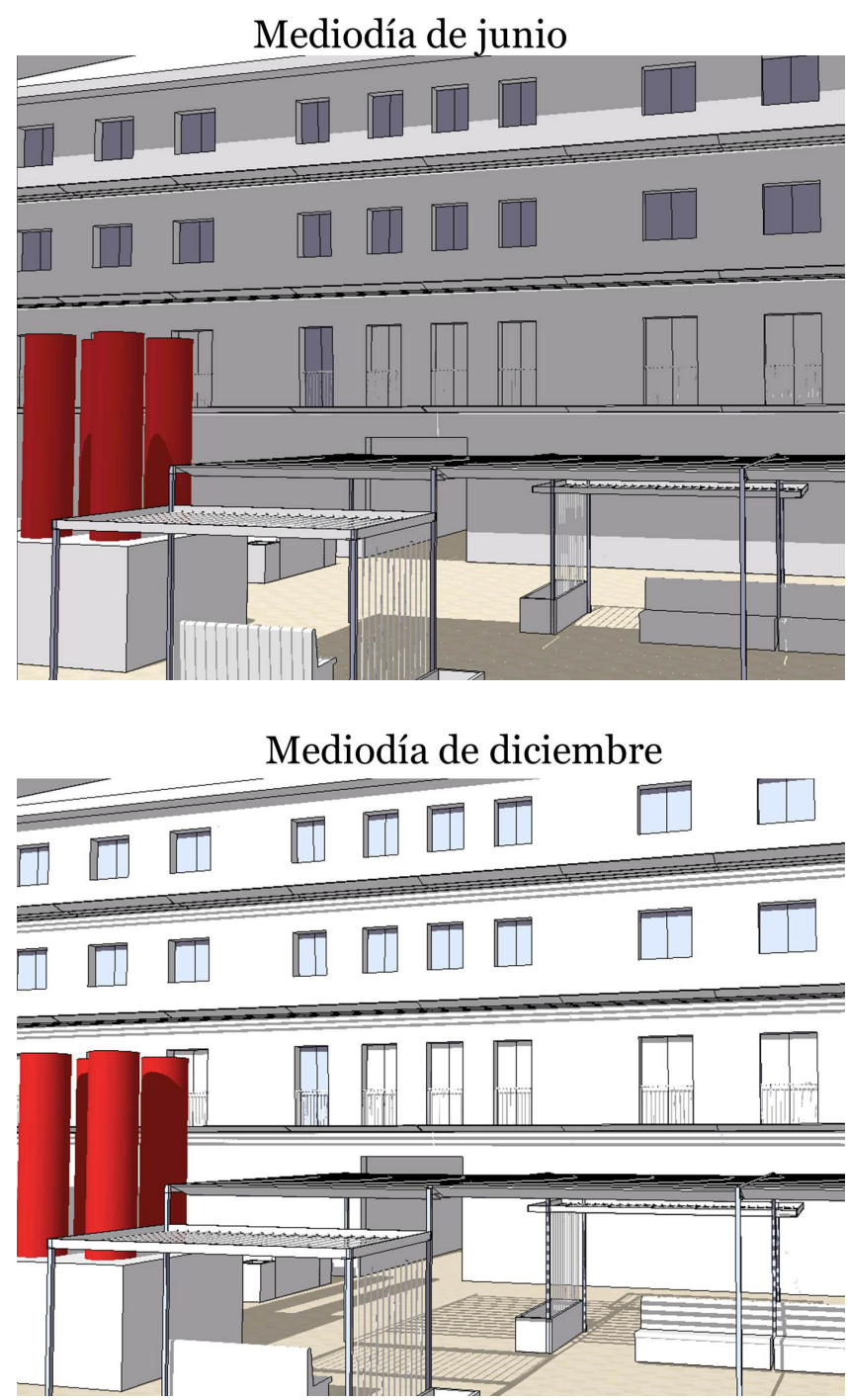

Figura 5. Efecto de sombreamiento y soleamiento de fachada sur con lamas fijas orientadas en edificio de viviendas (11). Fuente: Elaboración propia. 
Tabla 2. Tendencias de consumo energético según zonas climáticas y rehabilitación energética prevista para adaptarla a exigencias del CTE HE1 2006. Datos elaborados tomando como base el trabajo de M. de Luxán, G. Gómez, E. Roldán (9).

\begin{tabular}{|c|c|c|c|c|c|c|c|c|}
\hline \multicolumn{9}{|c|}{ Situación actual } \\
\hline \multicolumn{4}{|c|}{ Antes de intervención } & \multicolumn{5}{|c|}{ Después de intervención } \\
\hline CASO & \multicolumn{3}{|c|}{$\mathbf{A}$} & \multicolumn{4}{|c|}{ B } & \multirow[b]{2}{*}{$\begin{array}{c}\text { \% variación } \\
\text { respecto a la } \\
\text { situación actual }\end{array}$} \\
\hline $\begin{array}{c}\text { Zona } \\
\text { climática } \\
\text { actual }\end{array}$ & $\begin{array}{c}\text { CAEFC } \\
\left(\mathbf{k W h} / \mathbf{m}^{2}\right)\end{array}$ & $\begin{array}{c}\text { CAEFR } \\
\left(\mathbf{k W h} / \mathbf{m}^{2}\right)\end{array}$ & $\begin{array}{c}\text { CAEFCR } \\
\left(\mathrm{kWh} / \mathrm{m}^{2}\right)\end{array}$ & $\begin{array}{c}\text { Variación } \\
\text { CAEFC } \\
\left(\mathbf{k W h} / \mathbf{m}^{2}\right)\end{array}$ & $\begin{array}{c}\text { Variación } \\
\text { CAEFCR } \\
\left(\mathbf{k W h} / \mathbf{m}^{2}\right)\end{array}$ & $\begin{array}{c}\text { Variación } \\
\text { CAEFCR } \\
\left(\mathbf{k W h} / \mathbf{m}^{2}\right)\end{array}$ & $\begin{array}{c}\text { CAEFCR } \\
\left(\mathrm{kWh} / \mathbf{m}^{2}\right)\end{array}$ & \\
\hline $\mathrm{C} 1$ & 72,0 & o & 72,0 & $-15,8$ & $\mathrm{o}$ & $-15,8$ & 56,2 & $-21,9 \%$ \\
\hline D1 & 147,1 & o & 147,1 & $-15,1$ & 0 & $-15,1$ & 132,0 & $-10,2 \%$ \\
\hline $\mathrm{A} 3$ & 27,5 & 8,86 & 36,4 & $-15,7$ & $-4,3$ & $-20,0$ & 16,3 & $-55,0 \%$ \\
\hline \multicolumn{9}{|c|}{ Previsión cambio climático en 15 años } \\
\hline \multicolumn{4}{|c|}{ Antes de intervención } & \multicolumn{5}{|c|}{ Después de intervención } \\
\hline CASO & \multicolumn{3}{|c|}{$\mathbf{C}$} & D & & & & \multirow{2}{*}{$\begin{array}{c}\text { \% variación } \\
\text { respecto a la } \\
\text { situación actual }\end{array}$} \\
\hline $\begin{array}{c}\text { Zona } \\
\text { climática }\end{array}$ & $\begin{array}{c}\text { CAEFC } \\
\left(\mathbf{k W h} / \mathbf{m}^{2}\right)\end{array}$ & $\begin{array}{c}\text { CAEFR } \\
\left(\mathrm{kWh} / \mathbf{m}^{2}\right)\end{array}$ & $\begin{array}{c}\text { CAEFCR } \\
\left(\mathbf{k W h} / \mathbf{m}^{2}\right)\end{array}$ & $\begin{array}{c}\text { Variación } \\
\text { CAEFC } \\
\left(\mathbf{k W h} / \mathbf{m}^{2}\right)\end{array}$ & $\begin{array}{c}\text { Variación } \\
\text { CAEFCR } \\
\left(\mathbf{k W h} / \mathbf{m}^{2}\right)\end{array}$ & $\begin{array}{c}\text { Variación } \\
\text { CAEFCR } \\
\left(\mathbf{k W h} / \mathbf{m}^{2}\right)\end{array}$ & $\begin{array}{c}\text { CAEFCR } \\
\left(\mathbf{k W h} / \mathbf{m}^{2}\right)\end{array}$ & \\
\hline B3 & 30,9 & 12,6 & $-39,5 \%$ & $-3,3$ & $-6,1$ & $-9,4$ & 34,1 & $-52,6 \%$ \\
\hline $\mathrm{C} 2$ & 88,6 & 11,6 & $-31,9 \%$ & $-24,2$ & $-5,1$ & $-29,3$ & 70,8 & $-51,8 \%$ \\
\hline $\mathrm{A}_{4}$ & 17,8 & 11,9 & $-18,0 \%$ & $-9,4$ & $-5,6$ & $-15,0$ & 14,7 & $-59,3 \%$ \\
\hline
\end{tabular}

CAEFC Consumo anual de energía final en calefacción

CAEFR Consumo anual de energía final en refrigeración

CAEFCR Consumo anual de energía final en calefacción y refrigeración

Para ello es imprescindible un enfoque transversal y multidisciplinar, de manera que a la hora de definir las políticas de intervención para reducir el consumo energético de los edificios, se tengan en cuenta estudios e investigaciones de otras disciplinas, como la evaluación del impacto ambiental a largo plazo, la consideración de aspectos económicos y sociales que condicionan el comportamiento de los usuarios o su capacidad de adaptación en función del clima y del diseño de las viviendas. Así, será posible no solo proponer medidas más eficaces para el ahorro de energía y la mejora de la calidad de vida en las edificaciones, sino anticiparse a otras situaciones no deseables y plantear acciones de intervención adecuadas.
Esta cuestión tiene especial relevancia cuando el horizonte a medio plazo, marcado por las políticas europeas, hace especial énfasis en el consumo de energía nulo de los edificios y en la necesidad del fomento de una economía de bajo carbono. Para ello se necesita profundizar en el estudio del mayor número de factores que intervienen en la construcción y funcionamiento de las viviendas, así como la aplicación de nuevos enfoques disciplinares que permitan conocer a largo plazo la relación entre todos los elementos y garantizar que, con el paso del tiempo, se consiga un menor impacto en el medio ambiente.

\section{REFERENCIAS}

(1) IDAE. (2011). Proyecto Sech-Spahousec. Análisis del consumo energético del sector residencial en España, Madrid: Instituto para la Diversificación y Ahorro de Energía.

(2) León, A.L., Muñoz, S., León, J., Bustamante, P. (2010). Monitorización de variables medioambientales y energéticas en la construcción de viviendas protegidas: Edificio Cros-Pirotecnia en Sevilla. Informes de la Construcción, 62(519): 6782, doi: http://dx.doi.org/10.3989/ic.09.045.

(3) Boardman, B. (1991). Fuel Poverty: From Cold Homes to Affordable Warmth, London: Belhaven Press.

(4) Tirado Herrero, S., Jiménez Meneses, L., López Fernández, J.L., Martín García, J. (2014). Pobreza energética en España. Análisis de tendencias. Madrid: Asociación de Ciencias Ambientales.

(5) INE. (2012). Encuesta de Presupuestos Familiares Año 2012. Madrid: Instituto Nacional de Estadística. http://www. ine.es/prensa/epf_prensa.htm.

(6) INE. (2013). Encuesta de Condiciones de Vida. Base 2013. Madrid: Instituto Nacional de Estadística. http://www.ine. es/jaxi/menu.do?type=pcaxis\&path=/t25/p453\&file=inebase.

(7) AEMET. (2009). Generación de escenarios regionalizados de cambio climático para España. Madrid: Agencia Estatal de Meteorología. http://www.aemet.es/documentos/es/serviciosclimaticos/cambio_climat/datos_diarios/Informe_ Escenarios.pdf.

(8) Rodríguez-Villasante, T., Alguacil, K., Denche, C., Hernández Aja, A., León, C., Velázquez, I. (2009). Retrato de chabolista con piso. Análisis de redes sociales en la remodelación de barrios de Madrid. Madrid: ALFOZ-CIDUR/ IVIMASGV.

(9) Luxán, M. d., Gómez, G., Román, E. (2013, 21-22 de noviembre). Estrategias y posibilidades de un demostrador de rehabilitación de vivienda social, Proyecto Life New4OLD. En Jornadas internacionales de Investigación en Construcción. Vivienda: pasado, presente y futuro (p. 108). Madrid: Instituto de Ciencias de la Construcción Eduardo Torroja.

(10) Sánchez-Guevara, C., Román López, E., Gómez Muñoz, G. (2014, 11-13 de junio). Fuel poverty as a determinant in energy retrofitting actions. En 1st International Congress on research in Construction and Architectural Technologies. Madrid: Escuela Técnica Superior de Arquitectura de Madrid (ETSAM). 
(11) Proyecto LIFE New4Old (LIFE10 ENV/ES/439). Instituciones participantes: Centro Tecnológico AITEMIN, Universidad Politécnica de Madrid (UPM), Centro Tecnológico de la Cerámica y el Vidrio de Portugal(CTCV), Sociedad Municipal Zaragoza Vivienda (SMZV). http://www.newsolutions4oldhousing.eu/index.php?lang=es.

(12) INE. (1991). Censo de Población y Viviendas 1991. Madrid: Instituto Nacional de Estadística. http://www.ine.es/jaxi/ menu.do?type $=$ pcaxis\&path $=\% 2 \mathrm{Ft} 20 \% 2 \mathrm{Fe} 243 \&$ file $=$ inebase $\& \mathrm{~L}=0$.

(13) INE. (2007). Censo de Población y Viviendas de 2001. Madrid: Instituto Nacional de Estadística. http://www.ine.es/ censo2001/.

(14) Gómez Muñoz, G. (2014) Método de análisis diacrónico para la intervención en el alojamiento con criterios ecológicos. El caso de Madrid 1940-2100. (Tesis doctoral inédita). Escuela Técnica Superior de Arquitectura de Madrid (ETSAM). Universidad Politécnica de Madrid.

(15) MFOM. (2014). Número de licencias concedidas según tipo de obra. Madrid: Ministerio de Fomento.

(16) Luxán García de Diego, M. d., Vázquez Espí, M., Gómez Muñoz, G., Román López, E., \& Barbero Barrera, M. (2009). Actuaciones con criterios de sostenibilidad en la rehabilitación de viviendas en el centro de Madrid, Madrid: Empresa Municipal de la Vivienda y Suelo.

(17) Power, A. (2010). Housing and Sustainability: demolition or refurbishment. Urban and Design Planning, 163(4): 205216.

(18) Recaño, J. (2002). Proyección de Hogares de la Comunidad de Madrid 2002-2017. Madrid: Consejería de Economía e Innovación Tecnológica. http://www.madrid.org/iestadis/fijas/informes/descarga/proyhogares17me.pdf.

(19) Capdevila, I., Linares, E., Folch, R. (2012). Eficiencia energética en la rehabilitación de edificios, p. 42, 60, 70, 71, Barcelona: Fundación Gas Natural Fenosa, Consejo Superior de los Colegios de Arquitectos de España.

(20) Gómez Azpeitia, G., Bojórquez Morales, G., Ruiz Torres, R. (2007). El confort térmico: dos enfoques teóricos enfrentados. Palapa, 2(001): 44-57.

(21) Nicol, J., Humphreys, M. (2002). Adaptative thermal comfort and sustainable thermal standards for buildings. Energy and Buildings, 34: 563-572. doi: http://dx.doi.org/10.1016/So378-7788(02)oooo6-3.

(22) Luxán, M. d., Gómez, G., Barbero, M., Román, E. (2011). Estudio UPGRADE Confort para Energylab. (Sin publicar).

(23) Holmes, M., Hackner, J. (2007). Climate change, thermal comfort and energy: Meeting the design challenges of the 21st century, Energy and Buildings, 39(7): 802-814, doi: http://dx.doi.org/10.1016/j.enbuild.2007.02.009.

(24) Olcina Cantos, J. (2009). Cambio climático y riesgos climáticos en España, Investigaciones Geográficas, 49: 197-220.

(25) Vázquez Espí, M. (2001). Construcción e impacto sobre el ambiente: el caso de la tierra y otros materiales. Informes de la construcción, 52(471): 29-43, doi: http://dx.doi.org/10.3989/ic.2001.v52.i471.681. 